\title{
Síndrome de apnea del sueño y enfermedades cardiovaculares
}

\author{
C. ZAMARRÓN SANZ, J. GONZÁLEZ BARCALA, M. SALGUEIRO RODRÍGUEZ, \\ J.R. RODRÍGUEZ SUÁREZ
}

Unidad del Sueño. Servicio de Neumología. Hospital Clínico Universitario de Santiago. Santiago de Compostela. A Coruña

SLEEP APNEA SYNDROME AND CARDIOVASCULAR DISEASES

\section{RESUMEN}

El síndrome de apnea del sueño (SAS) es una enfermedad frecuente, reconocida como un problema de salud pública. Las enfermedades cardiovasculares son la causa de morbimortalidad más importante en estos pacientes, sin embargo los mecanismos subyacentes de esta asociación no están claramente establecidos.

Desde un punto de vista fisiopatológico en el SAS se producen diferentes fenómenos que pueden explicar la aparición de problemas cardiovasculares, tales como la hipoxia progresiva en relación con la apnea, los incrementos de la presión negativa intratorácica generada por los esfuerzos del sistema ventilatorio en contra de la vía aérea cerrada y las modificaciones en la actividad del sistema nervioso autónomo asociado con los microdespertares. Además, los episodios de hipoxia y reoxigenación existentes en el SAS pueden jugar un papel importante en la alteración del balance entre sustancias vasoconstrictoras y vasodilatadoras trastornando la hemostasis vascular y condicionando disfunción endotelial. Por otra parte, se ha descrito un incremento de la agregación plaquetaria y una disminución de la fibrinolisis en este grupo de pacientes, pudiendo ambos favorecer también la aparición de enfermedad vascular.

\section{ABSTRACT}

The sleep apnea syndrome is a common disease, recognised as a public health problem. Cardiovascular disease is the most frequent cau se of morbidity and mortality in these patients, however the underlying mechanisms of this association have not been clearly established.

In sleep apnea syndrome differents phenomena can be produced which may explain the appearance of cardiovascular problems, such a progressive hypoxia in relationship with the apnea, the increases of intrathoracic pressure cause by the efforts of ventilation system against close upper airway and the modifications of the autonomic nervous sys tem associated with the arousals. In addition, the hypoxia episodes and reoxigenation, which appear in the sleep apnea syndrome, may play a important role in the alteration of the balance between vasoconstriction and vasodilatation substances affecting the vascular homeostasis and conditioning endothelial dysfunction. On the other hand, the increasing of platelets aggregation and the decreased of fibrinolisis in this group of patients may cause vascular diseases.

KEY WORDS: Sleep apnea syndrome. Cardiovascular diseases. Endo- thelial dysfunction.
PALABRAS CLAVE: Síndrome de apnea del sueño. Enfermedad cardiovascular. Disfunción endotelial.

Zamarrón Sanz C, González Barcala J, Salgueiro Rodríguez M, Rodríguez Suárez JR. Síndrome de apnea del sueño y enfermedades cardiovaculares. An Med Interna (Madrid) 2001; 18: 274-279.

\section{INTRODUCCIÓN}

El síndrome de apnea del sueño (SAS) es una enfermedad común, con una prevalencia aproximada del $2 \%$ en mujeres y del $4 \%$ en hombres (1). Durante los últimos años, esta enfermedad ha sido reconocida como un problema importante de salud pública (2) y asociada a un incremento de morbimortalidad fundamentalmente de origen cardiovascular $(3,4)$.

El SAS presenta una compleja constelación de síntomas y signos, como consecuencia de la presencia de apneas recurrentes durante el sueño, debidas a la obstrucción parcial o completa de la vía aérea superior. Las apneas suelen finalizar cuando se produce un breve despertar o "arousal", con lo que los músculos dilatadores de la vía aérea superior recuperan el tono de vigilia y se reanuda el flujo. La repetición de la secuencia apneas - "arousals" durante toda la noche conduce a hipoxemia nocturna y fragmentación del sueño. Todo ello acarrea que el sueño del paciente con SAS sea diferente al normal, tanto desde un punto de vista cuantitativo como cualitativo.

La presencia de somnolencia diurna excesiva, ronquido y sueño agitado constituyen tres de las características clínicas principales del SAS. Además, estos pacientes pueden referir cefalea y sensación de embotamiento, fundamentalmente matutino.

El SAS se manifiesta en todas las edades y en ambos sexos, pero característicamente en varones de edad media, con ronquido habitual y sobrepeso $(1,5)$. Estudios realizados recientemente indican que esta enfermedad afecta al $6,8 \%$ de los sujetos entre 50 y 70 años (6).

Trabajo aceptado: 6 de Julio de 2000

Correspondencia: C. Zamarrón Sanz. Magdalena 4-4º A. Milladoiro. Ames. 15895 La Coruña. 
El diagnóstico de sospecha del SAS se establece básicamente a través de la historia clínica (5) sin embargo las técnicas de despistaje, como la oximetría, también pueden ayudarnos en la priorización de los estudios polisomnográficos (7). Esta prueba es fundamental para el diagnóstico definitivo e indispensable para el manejo terapéutico de los enfermos con SAS (8). El estudio polisomnográfico controla simultáneamente diferentes parámetros fisiológicos, que permiten evaluar la repercusión de las apneas sobre la función cardiorespiratoria y organización del sueño nocturno $\mathrm{y}$, en su caso, objetivar los efectos de la terapéutica aplicada. Además del análisis global del registro polisomnográfico, el cálculo del promedio de episodios de apnea e hipopnea por hora de sueño, o índice de apnea-hipopnea, es esencial para valorar la presencia de SAS.

Con respecto a la estrategia terapéutica utilizada, la pérdida de peso, la aplicación de presión positiva continua en la vía aérea (CPAP) y la cirugía son las tratamientos más empleados.

Las enfermedades del aparato circulatorio ocupan el primer lugar en la mortalidad por grandes grupos de causas en España, tanto en varones como en mujeres (9). Estas enfermedades son frecuentes en pacientes con SAS pero los mecanismos subyacentes de dicha asociación no son bien conocidos. El objetivo fundamental de esta revisión es el análisis de la asociación entre el SAS y la enfermedad cardiovascular.

\section{EL RONQUIDO Y LAS ENFERMEDADES CARDIOVASCULARES}

El ronquido habitual es un fenómeno frecuente con una prevalencia que oscila entre el 4 y el $29 \%$ en hombres y entre el 3 y el $14 \%$ en mujeres (10), incrementándose con la edad y asociándose con la obesidad y el hábito tabáquico (11). Aunque inicialmente considerado como un fenómeno anecdótico, actualmente está siendo objeto de intensas investigaciones con el objetivo de comprobar si este síntoma es nocivo para la salud del individuo. En este sentido, diferentes estudios epidemiológicos han mostrado su asociación a diversos factores de riesgo cardiovascular $(12,13)$, especialmente a la hipertensión arterial (14-17).

También se ha discutido la relación del ronquido con otras enfermedades cardiovasculares, como la cardiopatía isquémica. Inicialmente, en 1983, Partinen y cols. (18) habían observado que el $93 \%$ de los pacientes que ingresaban en una unidad de urgencias con infarto de miocardio presentaban ronquido habitual. Norton y Dunn (11) también constatan un incremento del riesgo de padecer enfermedad coronaria entre roncadores. D’Alessandro y cols. (1990) observan que el ronquido habitual está asociado significativamente al riesgo de desarrollar infarto de miocardio, de forma independiente del hábito tabáquico, hipertensión arterial, diabetes mellitus y consumo de alcohol (19). Seppäla y cols., (1991), en estudios necrópsicos, demostraron que la causa cardiovascular de muerte era más común entre aquéllos que roncaban habitualmente con respecto a los no roncadores y que el ronquido podía ser un factor de riesgo de muerte brusca durante la noche y las primeras horas de la mañana (20). Posteriormente, Smirne y cols. (1993) demuestran que el ronquido habitual es un factor de riesgo para enfermedad vascular aguda (21). Recientemente, en un estudio prospectivo de 4 años realizado en nuestro medio, el ronquido estaba significativamente relacionado con el desarrollo de cardiopatía isquémica (22).
La explicación fisiopatológica de la asociación entre el ronquido y la enfermedad cardiovascular puede basarse en que durante el ronquido se generan importantes esfuerzos inspiratorios en contra de un orificio faríngeo semicerrado, con importantes variaciones en la presión pleural, lo cual puede afectar a la precarga o a la postcarga cardíaca (23). Es posible que estos cambios, mantenidos en el tiempo, puedan favorecer el desarrollo de la enfermedad vascular (24).

Contrastando con lo expuesto anteriormente, otros autores no han encontrado asociación definitiva entre el ronquido y la enfermedad cardiovascular. Ello puede ser debido a la presencia de factores confusores como obesidad, edad, sexo, hábito tabáquico y alcohólico, o SAS subyacente no diagnosticado, presentes en los sujetos roncadores y en los pacientes con enfermedad cardiovascular, que pueden generar una falsa asociación entre ambos $(25,26)$.

\section{EL SAS Y LAS ENFERMEDADES CARDIOVASCULARES}

El SAS ha sido relacionado con complicaciones cardiovasculares (27-30). En diferentes estudios epidemiológicos se ha demostrado que alrededor de la mitad de los pacientes con SAS padecen hipertensión arterial y que la prevalencia de SAS en pacientes hipertensos es mayor que la existente en la población general $(27,31,32)$. También se ha comprobado la aparición de hipertensión arterial mantenida en modelos animales de síndrome de apnea del sueño (33).

Diferentes estudios muestran asociación entre el SAS y la cardiopatía isquémica. En 1982, De Olazábal señala que los enfermos con enfermedad coronaria presentan frecuentemente caídas en $\mathrm{la} \mathrm{SaO}_{2}$ y apneas durante el sueño (34). En un estudio de casos y controles realizado en Australia, Hung y cols. (1990) encuentran una asociación significativa entre SAS e infarto de miocardio, independiente-mente de la edad, índice de masa corporal, hipertensión arterial, hábito de fumar y nivel de colesterol sanguíneo, existiendo un incremento en el riesgo de infarto de miocardio a medida que se incrementan los niveles de índice apnea-hipopnea (35). Mooe y cols. (1996) en un estudios de casos y controles, también observan asociación entre el SAS y la enfermedad coronaria en ambos sexos $(36,37)$, hecho confirmado en otros trabajos más recientes $(38-40)$.

Sin embargo, al igual ocurre con el ronquido, la asociación entre el SAS y la enfermedad coronaria ha sido cuestionada argumentando diversos problemas metodológicos (41). Por todo ello, en los momentos actuales está en marcha un estudio prospectivo multicéntrico que probablemente pueda aportar datos definitivos (42).

MECANISMOS IMPLICADOS EN LA ASOCIACIÓN DE LA ENFERMEDAD VASCULAR CON EL SÍNDROME DE APNEA DEL SUEÑO

\section{ARTERIOSCLEROSIS Y ENFERMEDAD VASCULAR}

La arteriosclerosis es una patología muy prevalente en la sociedad occidental, y es el proceso fundamental de varias enfermedades, como la cardiopatía isquémica, enfermedad vascular cerebral o la arteriopatía obliterante periférica. El inicio y desarrollo de la placa aterosclerótica implicará el desequilibrio entre varios factores: entrada-salida de lípidos en la 
pared arterial, formación y destrucción de matriz colágena, todos ellos con diferentes influencias sobre el proceso inflamatorio que se produce en la placa. También influirán otros factores, que pueden ser metabólicos (hipercolesterolemia, diabetes mellitus), físicos (hipertensión arterial), químicos (tabaco y las grasas de la dieta) o genéticos. Cada uno de ellos podrá tener un papel más o menos relevante en los diferentes pasos de la patogenia de la arteriosclerosis (43) iniciándose dicho proceso con una disfunción endotelial antes de que exista un daño estructural del endotelio (44).

\section{DISFUNCIÓN ENDOTELIAL Y ENFERMEDAD VASCULAR}

Desde la pasada década, los aspectos dinámicos de la fisiología vascular, particularmente el papel del endotelio, han tenido una influencia decisiva en el entendimiento de la patología vascular. Esta monocapa celular que recubre el interior de los vasos, no solo tiene propiedades como barrera discriminatoria al paso de solutos, sino también secretoras. Entre estas destacan los factores vasodilatadores y vasoconstrictores derivados del endotelio, los cuales mantienen un equilibrio en condiciones fisiológicas. Cuando por una alteración ambiental se produce hiperactividad o hipoactividad funcional de uno $\mathrm{u}$ otro, aparece lo que conocemos como disfunción endotelial.

En el 1980, Furchgott demostró la producción de un factor relajante del endotelio (45), que posteriormente fue caracterizado como el óxido nítrico (46). Este compuesto es considerado el principal factor vasodilatador producido por el endotelio. Junto al óxido nítrico existen otros factores implicados en el proceso de disfunción endotelial como son la endotelina-1, un potente vasoconstrictor, diferentes moléculas de adhesión y algunos factores de coagulación y de fibrinolisis. En los momentos actuales, la medición de estos factores en sangre ha abierto una vía no invasiva para el estudio del proceso de disfunción endotelial en diferentes enfermedades, que puede ser útil para la clínica (47).

\section{INFLUENCIA DE LA DISFUNCIÓN ENDOTELIAL EN EL ORIGEN DE LA ENFERMEDAD VASCULAR}

La disfunción endotelial parece ser un evento inicial clave en la arteriosclerosis (48). El fallo de las respuestas vasodilatadoras, dependiente del endotelio, a estímulos fisiológicos es probable que condicionen una disminución de la producción o inactivación de óxido nítrico. En este sentido, existe evidencia experimental que demuestra que el óxido nítrico derivado del endotelio puede ser una molécula endógena antiateromatosa y que la reducción de la actividad de óxido nítrico puede facilitar la progresión de la arteriosclerosis (49). Además de la pérdida de la acción protectora del óxido nítrico, existen otros factores que pueden condicionar disfunción endotelial y progresión de la arteriosclerosis, como son el incremento de la secreción de sustancias vasoconstrictoras y la expresión de moléculas de adhesión de superficie.

Por otra parte, la elevación de los niveles de LDL puede inducir la aparición de todos los componentes de la reacción aterosclerótica, al acumularse dichas lipoproteínas en la pared arterial. En este sentido, las LDL oxidadas condicionan el reclutamiento de monocitos al inducir cambios en las moléculas que afectan los diferentes pasos de la unión monocitocélula endotelial (50-52).

\section{DISFUNCIÓN ENDOTELIAL Y SÍNDROME DE APNEA DEL SUEÑO}

El fenómeno de la disfunción endotelial se ha asociado con distintas enfermedades. En los años recientes, la implicación del endotelio ha sido ampliamente estudiada en la cardiopatía isquémica y en la hipertensión arterial. Se ha comprobado que el tono vascular coronario es dependiente de la producción endotelial de óxido nítrico $(53,54)$, habiéndose descrito en estos pacientes alteraciones en la respuesta vascular coronaria y en los niveles sanguíneos de los marcadores de disfunción endotelial (55-60).

Por otra parte es conocido que la hipertensión arterial participa fisiopatológicamente en la disfunción endotelial, alterando drásticamente el papel homeostático que ejerce el endotelio sobre la regulación de la función vascular (61-65). Además, existen datos en la literatura que apoyan la presencia de disfunción endotelial en pacientes con hipercolesterolemia (66-68), diabetes (69), tabaquismo (70,71) e insuficiencia renal (72).

Como se comentó previamente, diversos estudios epidemiológicos han demostrado asociación entre el SAS y las enfermedades cardiovasculares; siendo bien conocido que existen distintos mecanismos fisopatológicos que pueden determinar esta asociación. En el SAS, los episodios de apnea obstructiva condicionan un incremento de la presión negativa intratorácica, hipoxia progresiva, microdespertares y modifican la actividad del sistema nervioso autónomo $(27,73)$. Este cambio en la presión negativa intratorácica asociado a la apnea puede provocar la presencia de un estado de pseudohipervolemia, incremento del factor natriurético atrial y disminución de la actividad del eje renina-angiotensina-aldosterona $(74,75)$.

Por otra parte, el tono simpático está elevado en el SAS. Los registros neurofisiológicos y la cuantificación en plasma o en orina de catecolaminas o de sus metabolitos han demostrado la existencia de una activación simpática en estos pacientes (76-78), que se mantiene durante el día (79) y que revierte tras el tratamiento con $\operatorname{CPAP}(80,81)$. Este incremento global de la activación simpática puede inducir vasoconstricción y elevación mantenida de la presión arterial favoreciendo la aparición de cambios estructurales vasculares (79).

Diferentes datos de la literatura muestran que episodios de hipoxia y reoxigenación pueden distorsionar la hemostasis vascular, al modificar el balance entre sustancias vasoconstrictoras y vasodilatadoras (82-89). En estudios in vivo se ha observado un incremento de la secreción de endotelina en relación con la presencia de hipoxia (90). Estudios in vitro han demostrado que la expresión genética de la endotelina está incrementada durante la hipoxemia (91) y que existe un aumento de la secreción de esta sustancia en cultivos de células endoteliales sometidas a hipoxia (92).

Diferentes trabajos publicados recientemente muestran que los marcadores de disfunción endotelial están elevados en la sangre de los pacientes con SAS. Saarelainen y cols. (1997) cuantifican endotelina- 1 en el plasma de 23 pacientes con SAS y de 66 sujetos control comprobando la presencia de niveles sanguíneos más altos en el primer grupo. Diez casos fueron reestudiados después de tres meses de tratamiento con presión positiva continua de la vía aérea (CPAP) sin observar disminución de los niveles de este marcador (93).

Phillips y cols. (1999) estudian prospectivamente los cambios en la presión arterial, saturación arterial de oxígeno y niveles de endotelina- 1 circulante en 22 pacientes con SAS antes y después del tratamiento con CPAP. La presión arterial media y 
la concentración plasmática de endotelina-1 estaban incrementadas de forma significativa en sujetos con SAS y disminuyeron tras 5 horas de tratamiento con CPAP. Los cambios en los niveles de endotelina-1 se correlacionaron con los cambios de la presión arterial media y de la saturación arterial de oxígeno. Los autores concluyen que el SAS condiciona un incremento de los niveles sanguíneos de endotelina-1, que disminuye tras el tratamiento con CPAP. Además sugieren que el efecto vasoconstrictor de la endotelina puede estar implicado en el incremento del riesgo cardiovascular de los pacientes con SAS (94).

Ohga y cols., (1999) cuantificaron, en pacientes con SAS, los niveles circulantes de tres marcadores de disfunción endotelial (ICAM-1, VCAM-1 y L-selectina) antes y después del sueño, aparejados por edad con un grupo de sujetos control. Los niveles de estas sustancias estaban incrementados en pacientes con SAS antes del sueño comparado con los controles. Después del sueño, las diferencias observadas anteriormente se hacían más marcadas. Los autores concluyen que el SAS induce activación de algunos marcadores implicados en la disfunción endotelial a través de la hipoxia, condicionando un incremento del riesgo cardiovascular (95).

Rangemark y cols., (1995) estudiaron la función plaquetaria y la actividad fibrinolítica de 13 sujetos con SAS y 10 sujetos controles, aparejados por sexo y peso. El inhibidor del activador del plasminógeno tipo 1 (PAI-1) presentaba unos niveles superiores en el grupo con SAS con respecto al control, indicando una disminución en la actividad fibrinolítica, lo cual puede facilitar la aparición de enfermedades vasculares en estos pacientes (96). Otros autores como Bokinsky y cols., (1995) observaron un incremento de la activación y agregación plaquetarias en estos pacientes y que este efecto disminuía con la CPAP (97).

Independientemente de los datos anteriores, existen otras hipótesis que pueden ser consideradas a la hora de explicar la alta prevalencia de enfermedades cardiovasculares en los pacientes con SAS. En este sentido, es posible que los sucesivos episodios de hipoxemia- reoxigenación relacionados con la apnea, además de alterar la relación entre vasoconstricción y vasodilatación, puedan contribuir a la oxidación de las lipo- proteínas de baja densidad y aumentar su potencial efecto aterogénico (98-101).

Wali y cols. (1998) estudiaron la susceptibilidad al estrés oxidativo de las LDL en 15 pacientes con SAS (6 con más de 10 minutos de registro con $\mathrm{SaO}_{2}<85 \%$ ) y 9 sujetos con SAS sin el criterio anterior (no hipóxicos). Estos autores no encontraron diferencias entre ambos grupos por lo que concluyen que las LDL de los pacientes con SAS y desaturaciones importantes durante el sueño no presentan LDL más susceptibles a la oxidación que la de los pacientes con SAS y desaturaciones menos importantes (102).

Saarelainen y cols. (1999) cuantificaron los anticuerpos contra LDL oxidadas en 297 pacientes con SAS y 54 controles presentando los primeros unos niveles de anticuerpos superiores al de los controles (ajustando por edad, BMI y sexo). Los autores proponen a esta técnica como un indicador de disfunción endotelial así como una herramienta de trabajo para el despistaje de sujetos con SAS y con riesgo importante para el desarrollo de enfermedad cardiovascular (103).

Por todo lo anteriormente referido, la disfunción endotelial se caracteriza por un aumento del tono vasoconstrictor, un incremento de la adhesión de leucocitos, mayor tendencia a la trombosis y a la agregación plaquetaria, junto a la proliferación y migración de las células musculares lisas. En el SAS se ha encontrado un incremento de los niveles de endotelina, moléculas de adhesión, aumento de la agregabilidad plaquetaria y disminución de la fibrinolisis, todos ellos marcadores de disfunción endotelial. Además es posible que estos pacientes presenten un aumento global de la activación simpática y mayores niveles de LDL oxidadas. Todos estos factores pueden facilitar el desarrollo de enfermedad vascular y explicar la mayor prevalencia de la misma en el SAS. Sin embargo, este hecho no ha sido suficientemente estudiado ya que los trabajos publicados hasta el momento se basan en un escaso número de sujetos y la influencia de la terapéutica con CPAP no ha sido claramente evaluada. El estudio de este problema será importante para el entendimiento de la patogénesis de las enfermedades vasculares en el SAS lo que permitirá guiar la terapéutica apropiada.

\section{Bibliografía}

1. Young T, Platt M, Dempsey J, Skatrud J, Weber S, Badr S. The occurrence of sleep disordered breathing among middle-aged adults. $\mathrm{N}$ Engl J Med 1993; 328: 1230-1235.

2. Phillipson EA. Sleep apnea. A major public health problem. N Engl J Med 1993; 328: 1271-1273.

3. He J, Kryger MH, Zorick FJ, Conway W, Roth T. Mortality and apnea index in obstructive sleep apnea. Chest 1988; 94: 9-14.

4. Partinen M, Jamieson A, Guilleminault C. Long-term outcome for obstructive sleep apnea syndrome patients. Mortality. Chest 1988; 94: 1200-1204.

5. Zamarrón C, Gude F, Otero Y, Alvarez Dobaño JM, Golpe A, Rodriguez Suárez JR. Sintomatología del síndrome de apnea del sueño en población general. Arch Bronconeumol 1998; 34: 245-249.

6. Zamarrón C, Gude F, Oero Y, Alvarez JM, Golpe A, Rodriguez JR. Prevalence of sleep disordered breathing and sleep apnea in 50 to 70 years old individuals. A survey. Respiration 1999; 66: 317-322.

7. Zamarrón C, Romero PV, Rodriguez JR, Gude F. Oximetry spectral analysis in the diagnosis of obstructive sleep apnea. Clin Sci (Colch) 1999; 97: 467-473.
8. American Thoracic Society. Indications and standards for cardiopulmonary sleep studies. Am Rev Respir Dis 1989; 139: 559-568.

9. Banegas Banegas JR, Villar Álvarez F, Martín Moreno JM, Rodríguez Artalejo F, González Enríquez J. Relevancia de la mortalidad por enfermedades del aparato circulatorio en España. Rev Clin Esp 1992; 190: 321-327.

10. Waller PC, Bhopal RS. Is snoring a cause of vascular disease?. An epidemiological review. Lancet 1989; 21: 143-146.

11. Norton PG, Dunn EV. Snoring as a risk factor for disease: An epidemiological survey. Br Med J 1985; 291: 630-621.

12. Jennum P, Hein HO, Suadicani P, Gyntelberg F. Cardiovascular risk factors in snores. A cross-sectional study of 3223 men aged 54 to 74 years: The Copenhagen male study. Chest 1992; 102: 1371-1376.

13. Zamarrón C, Gude F, Otero Y, Alvarez Dobaño JM, Salgueiro Rodríguez M, Rodríguez Suárez JR. El ronquido y los factores de riesgo vascular. Una encuesta epidemiológica. Neumosur 1998;10: 155-158.

14. Gislason T, Abert H, Taube A. Snoring and systemic hypertension and epidemiological study. Acta Med Scand 1987; 222: 415-421. 
15. Partinen M, Kaprio J, Koskenvuo M, Langinvainio H. Snoring and hypertension: A cross-sectional study on 12808 Finns aged 24-65 years. Sleep Res 1983; 12: 273.

16. Gislason T, Benediktsdottir B, Bjornsson J, Kjartansson G, Kjeld M, Kristbjarnarson H. Snoring, hypertension, and the sleep apnea syndrome. An epidemiological survey of middle-ages women. Chest 1993; 103: 1147-1151.

17. Hla K, Young T, Bidwell T, Palta M, Skatrud J, Dempsey J. Sleep apnea and hypertension. A population-based study. Ann Intern Med 1994; 120: 382-388.

18. Partinen M, Alikanka J, Lang H, Kaliomaki L. Myocardial infarction in relation to sleep apnea. Sleep Res 1983; 12: 272.

19. D'Alessandro R, Magelli C, Gamberini G, Bachelli S, Cristina E, Magnani B, Lugaresi E. Snoring every night as a risk factor for myocardial infarction: A case-control study. Br Med J 1990; 300: 1557-1558.

20. Seppälä T, Partinen M, Penttilä A, Aspholm R, Tiainen E, Kaukianen, A. Sudden death and sleeping history among Finnish men. J Intern Med 1991; 229: 23-28.

21. Smirne S, Palazzi S, Zucconi M, Chierchia S, Ferini-Strambi L. Habitual snoring as a risk factor for acute vascular disease. Eur Respir J 1993; 6: 1357-1361.

22. Zamarron C, Gude F, Otero Otero Y, Rodriguez Suárez JR. Snoring and myocardial infarction: a 4-year follow-up study. Respir Med 1999; 93: $108-112$.

23. Stradling JR, Crosby JH. Relation between systemic hypertension and sleep hypoxaemia or snoring: analysis in 748 men drawn for general practice. Br Med J 1990; 300: 75-78.

24. Ketterer MW, Brymer J, Rhoads K, Kraft P, Kenyon L. Snoring and the severity of coronary artery disease in men. Pychosom Med 1994; 56: 232-236.

25. Shmidt-Nowara WW, Coultas DB, Wiggins C, Skipper BE, Samet MS. Snoring in a Hispanic-American population. Risk factors and association with hypertension and other morbility. Arch Intern Med 1990; 150: 597-601.

26. Hoffstein V. Blood pressure, snoring, obesity, and nocturnal hypoxaemia. Lancet 1994; 344: 643-645.

27. Fletcher EC. The relationship between systemic hypertension and obstructive sleep apnea: Facts and theory. Am J Med 1995; 98: 118-128.

28. Zamarrón C, Salgueiro M, Alvarez JM, Otero Y, Rodríguez Suárez JR. Enfermedades cardiovasculares más frecuentes y síndrome de apnea del sueño. Un estudio en población general. An Med Intern Madrid 1998 15: 142-144.

29. Young T, Peppard P, Palta M, Hla KM, Finn L, Morgan B, Skatrud J. Population based study of sleep disordered breathing as a risk factor for hypertension. Arch Intern Med 1997; 157: 1746-1752.

30. Weiss JW, Launois SH, Anand A, Garpestad E. Cardiovascular morbidity in obstructive sleep apnea. Prog Cardiovasc Dis 1999; 41: 367-376.

31. Williams AJ, Houston D, Finberg S, Lam C, Kinney JL, Santiago S. Sleep apnea syndrome and essential hypertension. Am J Cardiol 1985; 55: 1019-1022.

32. Millman RP, Redline S, Carlisle CC, Assaf AR, Levinson PD. Daytime hypertension in obstructive sleep apnea. Chest 1991;99: 861-866.

33. Brooks D, Horner RL, Kozar LF, Render Teixeira CL, Phillipson EA. Obstructive sleep apnea as a cause of systemic hypertension. Evidence from a canine model. J Clin Invest 1997; 99: 106-109.

34. De Olazábal JR, Miller MJ, Cook WR, Mithoefer JC. Disorders breathing and hipoxia during sleep in coronary artery disease. Chest 1982; 82: $548-552$.

35. Hung J, Whitford EG, Parsons EW, Hillman DR. Association of sleep apnea with myocardial infarction in men. Lancet 1990; 336: 261-264.

36. Mooe T, Rabben T, Wiklund U, Franklin KA, Eriksson P. Sleep disordered breathing in women: Occurrence and association with coronary artery disease. Am J Med 1996; 101: 251-256.

37. Mooe T, Rabben T, Wiklund U, Franklin KA, Eriksson P. Sleep disordered breathing in men with coronary artery disease. Chest 1996; 109: 659-663.

38. Peker Y, Kraiczi H, Hedner J, Loth S, Johansson A, Bende M. An independent association between obstructive sleep apnoea and coronary artery disease. Eur Respir J 1999; 14: 179-184.

39. Aboyans V, Lacroix P, Virot P, Tapie P, Cassat C, Rambaud G, Laskar $\mathrm{M}$, Bensaid J. Sleep apnoea syndrome and the extent of atherosclerotic lesions in middle-aged men with myocardial infarction. Int Angiol 1999; 18: 70-73.

40. Zamarrón C, Amaro A, Fernandez F, Gude F, Mazaira A, Rodriguez Suarez JR. Sleep apnea syndrome and ischemic heart disease. An epidemiological study. Am J Respir Crit Care Med 1999; 159: 529.
41. Wright J, Johns R, Watt I, Melville M, Sheldon T. Health effects of obstructive sleep apnoea and the effectiveness of continuous positive airways pressure: a systematic review of the research evidence. BMJ 1997; 314: 851-860.

42. Quan SF, Howard BV, Iber C, Kiley JP, Nieto FJ, O’Connor GT, Rapoport DM, Redline S, Robbins J, Samet JM, Wahl PW. The Sleep Heart Study: Design, Rationale, and Methods Sleep; 20: 1077-1085.

43. Fuster V, Badimon L, Badimon JJ, Chesebro JH. The pathogenesis of coronary artery disease and the acute coronary syndromes. N Engl J Med 1992; 326: 242- 250.

44. Fuster V, Lewis A. Corner Memorial Lecture. Mechanisms leading to myocardial infarction: insights from studies of vascular biology. Circulation 1994; 90: 2126-2146.

45. Furchgott RF, Zawadski JV. The obligatory role of endothelial cells in the relaxation of arterial smooth muscle by acetylcholine. Nature 1980; 288; 373-376.

46. Palmer RM, Ferrige AG, Moncada S. Nitric oxide release accounts for the biological activity of endothelium-derived relaxing factor. Nature 1987; 327: 524-526.

47. Mullen MJ, Thorne SA, Deanfield JE. Non invasive assessment of endothelial function. Heart 1997; 77: 297-298.

48. Berliner JA, Navab M, Fogelman AM, Frank JS, Demer LL, Edwards PA, Watson AD, Lusis AJ. Atherosclerosis: basic mechanisms oxidation, inflammation, and genetics. Circulation 1995; 91: 2488-2496.

49. Cooke JP, Tsao PS. Is NO an endogenous antiatherogenic molecula? Arterioscler Thromb 1994; 14: 753-759.

50. Ross R. The pathogenesis of atherosclerosis: a perspective for the 1990s. Nature 1993; 362: 801-809.

51. Poston RN, Haskard DO, Coucjer JR, Gall NP, Johnson-Tidey RR. Expression of intercellular adhesion molecule-1 in atherosclerotic plaques. Am J Pathol 1992; 140: 665-673.

52. Libby P. Molecular bases of the acute coronary syndromes. Circulation 1995; 91: 2844-2850.

53. Jones CJH, Kuo L, Davis MJ, Defily DV, Chilian WM. Role of nitric oxide in coronary microvascular responses to adenosine and increased metabolic demand. Circulation 1995; 91: 1807-1813.

54. Kawai C. Pathogenesis of acute myocardial infarction. Novel regulatory systems of bioactive substances in the vessel wall. Circulation 1994; 90: 1033-1043.

55. Gordon JB; Ganz P, Nabel EG, Fish RD, Zebede J, Mudge GH, Alexander RW, Selwyn AP. Atherosclerosis influences the vasomotor response of epicardial coronary arteries to exercise. J Clin Invest 1989; 83: 1946-1952.

56. Ray SG, McMurray JJ, Morton JJ, Dargie HJ. Circulating endothelin in acute ischaemic syndromes. Br Heart J 1992; 67: 383-388.

57. Ikeda H, Nakayama H, Oda T, Kuwano K, Muraishi A, Sugi K, Koga Y, Toshima H. Soluble form of P-selectin in patients with acute myocardial infarction. Coronary Artery Dis 1994, 5: 515-518.

58. Lip GY, Blann AD. Von Willebrand factor and its relevance to cardiovascular disorders. Br Heart J 1995; 74: 580-583.

59. Blann AD, Amiral J, McCollum CN. Circulating endotelial cell/leucocyte adhesion molecules in ischaemic heart disease. Br J Haematol 1996; 95: 263-265.

60. Ridker PM, Hennekens CH, Roitman-Johnson B, Stampfer MJ, Allen J. Plasma concentration of soluble intercellular adhesión molecule 1 and risks of future myocardial infarction in apparently healthy men. Lancet 1998; 351: 88-92.

61. Panza JA, Quyyumi AA, Brush JE Jr, Epstein SE. Abnormal endothelium-dependent vascular relaxation in patients with essential hypertension N Engl J Med 1990; 323: 22-27.

62. Saito Y, Nakao K, Mukoyama M, Imura N. Increased plasma endothelin level in patients with essential hypertension. N Engl J Med 1990; 332: 205.

63. Vanhoutte PM. Endothelial dysfunction in hypertension. J Hypertens 1996; 14: S83-93.

64. Nava E, Farre AL, Moreno C, Casado S, Moreau P, Cosentino F, Luscher TF. Alterations to the nitric oxide pathway in the spontaneously hypertensive rat. J Hypertens 1998; 16: 609-615.

65. Ferro CJ, Webb DJ. Endothelial dysfunction and hypertension. Drugs 1997; 53: 30-41.

66. Lerman A, Webster MW, Chesebro JH, Edwards WD, Wei C, Fuster V, Burnett JC. Circulating and tissue endothelin immunoreactivity in hypercholesterolemic pigs. Circulation 1993; 88; 2923-2928.

67. Sorensen KE, Celermajer DS, Georgakopoulos D, Hatcher G, Betteridge DJ, Deanfield JE. Impairment of endothelium-dependent dilation is an early event in children with familial hypercholesterolemia and is related to the lipoprotein (a) level. J Clin Invest 1994; 93: 50-55. 
68. Hackman A, Abe Y, Insull W Jr, Pownall H, Smith L, Dunn K, Gotto AM Jr, Ballantyne CM. Levels of soluble cell adhesion molecules in patients with dislipidemia. Circulation 1996; 93: 1334-1338.

69. Goodfellow J, Ransey M, Luddington L, Jones C, Coates P, Dunstan F, Lewis MJ, Owens DR, Henderson AH. Endothelium and inelastic arteries: an early marker of vascular dysfunction in non insulin depended diabetes. BMJ 1996; 312: 744-745.

70. Celermajer DS, Sorensen KE, Gooch VM, Soiegelhalter DJ, Miller OI, Sullivan ID, Lloyd JK, Deanfield JE. Non invasive detection of endothelial dysfunction in children and adults at risk of atherosclerosis. Lancet 1992; 340: 1111-1571.

71. Drexler H, Hornig B. Endothelial dysfunction in human disease. J Mol Cell Cardiol 1999; 31: 51-60.

72. Koyama N, Tabata T, Nishzawa Y, Inoue T, Morii H, Yamaji T. Plasma endothelin levels in patients with uraemia. Lancet 1989; 1: 991-992.

73. McNamara SG, Brunstein RR, Sullivan CE. Obstructive sep apnoea. Thorax 1993; 48: 754-764.

74. Mackay TW, Fitzpatrick MF, Freestone S, Lee MR, Douglas NJ Atrial natriuretic peptide levels in the sleep apnoea/hypopnoea syndrome. Thorax 1994; 49: 920-921.

75. Hedner J. Vascular function in OSA. Sleep 1996; 19: 213-217.

76. Carlson JT, Hedner J, Elam M, Ejnell H, Sellgren J, Wallin BG. Augmented resting sympathetic activity in awake patients with obstructive sleep apnea. Chest 1993; 103: 1763-1768.

77. Coy TV, Dimsdale JE, Ancoli-Israel S, Clausen. Sleep apnoea and sympathetic nervous system activity: a review. J Sleep Res 1996; 5: 42-50.

78. Narkiewicz K, van de Borne PJ, Cooley RL, Dyken ME, Somers VK. Sympathetic activity in obese subjects with and without obstructive sleep apnea. Circulation 1998; 98: 772-776.

79. Noda A, Yasuma F, Okada T, Yokota M. Circadian rhythm of autonomic activity in patients with obstructive sleep apnea syndrome. Clin Cardiol 1998; 21: 271-276.

80. Hedner J, Darpo B, Ejnell H, Carlson J, Caidahl K. Reduction in sympathetic activity after long-term CPAP treatment in sleep apnoea: cardiovascular implications. Eur Respir J 1995; 8: 222-229.

81. Narkiewicz K, Kato M, Phillips BG, Pesek CA, Davison DE, Somers VK. Nocturnal continuous positive airway pressure decreases daytime sympathetic traffic in obstructive sleep apnea. Circulation 1999; 100: 2332-2335.

82. Lüscher TF. Endothelin: systemic arterial and pulmonary effects of a new peptide with potent biological properties. Am Rev Respir Dis 1992; 146: 56-60.

83. Inauen W, Granger DN, Meininger CJ, Schelling ME, Granger HJ, Kvietys PR. Anoxia-reoxygenation induced, neutrophil mediated endothelial cell injury: role of elastase. Am J Physiol 1990; 259: 925-931.

84. Wiles ME, Hechtman HB, Morel NM, Shepro D. Hypoxia reoxygenation induced injury of cultured pulmonary microvessel endothelial cells. Leukoc Biol 1993; 53: 490-497.

85. Yoshida N, Granger DN, Anderson DC, Rothlein R, Lane C, Kvietys PR. Anoxia - reoxygenation induced neutrophil adherence to cultured endothelial cells. Am J Physiol 1992; 262: 1891- 898.

86. Yokoyama S, Korthuis RJ, Benoit JN. Hypoxia reoxygenation impairs endothelium dependent relaxation in isolated rat aorta. Am J Physiol 1996; 270: 1126-1131.
87. Fike CD, Kaplowitz MR, Thomas CJ, Nelin LD. Chronic hypoxia decreases nitric oxide production and endothelial nitric oxide synthase in newborn pig lungs. Am J Physiol 1998; 274: 517-526.

88. Baker JE, Holman P, Kalyanaraman B, Griffith OW, Pritchard KA Jr. Adaptation to chronic hypoxia confers tolerance to subsequent myocardial ischemia by increased nitric oxide production. Ann N Y Acad Sci 1999; 874: 236-253.

89. Muramatsu M, Oka M, Morio Y, Soma S, Takahashi H, Fukuchi Y. Chronic hypoxia augments endothelin $\mathrm{B}$ receptor mediated vasodilation in isolated perfused rat lungs. Am J Physiol 1999; 276: 358-364.

90. Horio T, Kohno M, Yokokawa K, Murakawa K, Yasunari K, Fujiwara H, Kurihara N, Takeda T. Effect of hypoxia on plasma immunoreactive endothelin-1 concentration in anesthetized rats. Metabolism 1991; 40: 999-1001.

91. Kourembanas S, Marsden PA, Mcquillan LP, Faller DV. Hipoxia induces endothelin gene expresion and secretion in cultured human endothelium. J Clin Invest 1991; 88: 1054-1057.

92. Hieda HS, Gomez Sanchez CE. Hypoxia increases endothelin release in bovine endothelial cells in culture, but epinephrine, norepinephrine, serotonin, histamine and angiotensin II do not. Life Sci 1990; 47: 247-251.

93. Saarelainen S, Seppala E, Laasonen K, Hasan J. Circulating endothelin 1 in obstructive sleep apnea. Endothelium 1997; 5: 115-118.

94. Phillips BG, Narkiewicz K, Pesek CA, Haynes WG, Dyken ME, Somers VK. Effects of obstructive sleep apnea on endothelin-1 and blood pressure. J Hypertens 1999; 17: 61- 66.

95. Ohga E, Nagase T, Tomita T, Teramoto S, Matsuse T, Katayama H, Ouchi Y. Increased levels of circulating ICAM-1, VCAM-1, and L-selectin in obstructive sleep apnea syndrome. Appl Physiol 1999; 87: 10-14.

96. Rangemark C, Hedner JA, Carlson JT, Gleerup G, Winther K. Platelet function and fibrinolytic activity in hypertensive and normotensive sleep apnea patients. Sleep 1995; 18: 188-194.

97. Bokinsky G, Miller M, Ault K, Husband P, Mitchell J. Spontaneous platelet activation and aggregation during obstructive sleep apnea and its response to therapy with nasal continuous positive airway pressure. A preliminary investigation. Chest 1995; 108: 625-630.

98. Weisser B, Locher R, Mengden T, Sachinidis A, Vetter W Oxidized low-density lipoproteins in atherogenesis: possible mechanisms of action. J Cardiovasc Pharmacol 1992; 19: 4-7.

99. Dean RT, Wilcox I Possible atherogenic effects of hypoxia during obstructive sleep apnea. Sleep 1993; 16: 15-21.

100. Balagopalakrishna C, Nirmala R, Rifkind JM, Chatterjee S. Modification of low density lipoproteins by erythrocytes and hemoglobin under hypoxic conditions. S Adv Exp Med Biol 1997; 411: 337-345.

101. Holvoet P, Collen D Oxidation of low-density lipoproteins in the pathogenesis of atherosclerosis. Atherosclerosis 1998;137: 33-38.

102. Wali SO, Bahammam AS, Massaeli H, Pierce GN, Iliskovic N, Singal PK, Kryger MH. Susceptibility of LDL to oxidative stress in obstructive sleep apnea. Sleep 1998; 21: 290-296.

103. Saarelainen S, Lehtimaki T, Jaakkola O, Poussa T, Nikkila M, Solakivi T, Nieminen MM. Auto antibodies against oxidised low-density lipoprotein in patients with obstructive sleep apnoea. Clin Chem Lab Med 1999; 37: 517-520. 